\title{
Aptitud clínica ante el paciente pediátrico con asma grave en residentes de pediatría y urgencias
}

\author{
J. Loría-Castellanos, G. Márquez-Ávila, M.A. Valladares-Aranda
}

Objetivo. Construir, validar y aplicar un instrumento para evaluar la aptitud clínica de los residentes de pediatría y urgencia ante los pacientes pediátricos con asma grave. Sujetos y métodos. Estudio observacional autorizado por el comité de investigación. El instrumento se construyó a través de cinco casos clínicos reales, validado por consenso de expertos en pediatría, urgencias e investigación educativa, obteniéndose una versión final de 150 ítems con una consistencia de 0,89 . Se evaluaron 10 residentes de pediatría y 21 de urgencias. Se realizó un análisis estadístico no paramétrico. Resultados. La mayoría de los residentes $(79,73 \%)$ se ubicaron en los niveles bajos-muy bajos de aptitud clínica, ninguno alcanzó niveles superiores. Las pruebas estadísticas no encontraron diferencias entre los grados académicos o la especialidad. Conclusiones. El instrumento construido tiene una adecuada consistencia. El proceso educativo al que se han sometido estos residentes parece no favorecer el desarrollo de reflexión, lo que podría limitar su práctica profesional real.

Palabras clave. Aptitud clínica. Asma. Educación. Residentes.

\section{Clinical skills at the pediatric patient with severe asthma of Pediatrics and Emergency residents}

Aim. To develop, validate and implement a tool to assess the clinical competence of pediatric residents and medical emergencies to pediatric patients with severe asthma. Subjects and methods. An observational study approved by the research committee. The instrument was built through five problematized clinical cases, validated by consensus by experts in pediatrics, emergency and educational research, obtaining a final version of 150 items with a consistency of 0.89 . It evaluated 10 pediatric residents and 21 of emergency. We performed a nonparametric statistical analysis Results. Most residents (79.73\%) were located in low-very low levels of clinical competence, none reached higher levels. Statistical tests found no differences between academic degrees or specialty Conclusions. The educational process that these students have had seems to favor the development of reflection, which could limit its actual practice.

Key words. Asthma. Clinical skills. Education. Residents.

\section{Introducción}

El asma es una enfermedad de elevada morbimortalidad que se conoce desde hace más de 2.000 años y que en la actualidad constituye un problema de salud pública de ámbito mundial [1,2].

Entre diversos elementos que han dificultado la interpretación de los datos disponibles respecto del asma destacan la poca precisión en su definición y diagnóstico [3]. Aunque se han empleado a lo largo del tiempo diferentes definiciones, actualmente la más reconocida es la generada por los expertos de la Global Initiative for Asthma (GINA) y del Estudio Internacional sobre Asma y Alergias de la Infancia -International Study of Asthma and Allergy in Childhood (ISSAC)-, los cuales establecen que el asma es un problema inflamatorio crónico de las vías aéreas en el que intervienen múltiples células y mediadores de la inflamación, lo que ocasiona la obstrucción reversible del flujo aéreo y que se manifiesta con cuadros recurrentes de tos, disnea, sibilancias y sensación de opresión torácica, principalmente por la noche y la madrugada [4-8].
Servicio de Urgencias. Hospital General Regional 25. Instituto Mexicano del Seguro Social. Iztapalapa, México.

\section{Correspondencia} Dr. Jorge Loría Castellanos. Avda. Central 175. Barcelona 505. San Pedro de los Pinos. Del. Álvaro Obregón. CP 01180. México DF, México.

E-mail jloriac@hotmail.com 
En México se tiene información limitada sobre la prevalencia y comportamiento del asma, dentro del esquema del ISAAC. Romieu et al comunicaron la prevalencia acumulada de asma por diagnóstico médico y por sibilancias del 5,8 y $21,8 \%$, respectivamente; la prevalencia de sibilancias en los últimos 12 meses fue del $8,9 \%$ en el grupo de 6-8 años de edad, y del 6,6\% en el grupo de 11-14 años $[9,10]$. Los síntomas graves de asma fueron más notables en el grupo de 6-8 años y tendieron a predominar durante los meses de otoño e invierno.

En el estudio para evaluar los efectos de la contaminación en escolares del área metropolitana de la Ciudad de México se encontró una prevalencia de diagnóstico médico del 4,5\% y una prevalencia de sibilancias, acompañadas de dificultad respiratoria, del 8,3\% [11,12].

El asma en el niño se ha clasificado en diferentes tipos, lo que tiene importancia para decidir el esquema de tratamiento, para comparar diversas series clínicas y con fines pronósticos. A continuación se detalla la clasificación más aceptada [4-8]:

- Asma intermitente, episódica. Síntomas leves desencadenados por alérgenos o infecciones respiratorias víricas. En algunos niños se presenta con tos nocturna o inducida por ejercicio. Pueden existir semanas o meses sin síntomas entre estos episodios.

- Asma persistente leve. Después de que los primeros episodios se hayan desencadenado por infecciones respiratorias, a la edad de 5-6 años estos pacientes presentan cuadros desencadenados por diversos estímulos. En general, los síntomas están presentes por la noche y producen alteración significativa en las actividades normales del niño. Los episodios suelen aparecer varias veces al mes y son generalmente leves.

- Asma persistente moderada. Es similar a la anterior, pero con mayor gravedad durante las crisis, que se presentan varias veces a la semana y requieren consultas a servicios de urgencias y, ocasionalmente, hospitalización.

- Asma persistente grave. El niño presenta sintomatología de forma diaria, con alteración importante de sus actividades normales, en especial relacionadas con el sueño y las actividades físicas. Frecuentemente estas crisis requieren atención de urgencia y hospitalizaciones, incluso en unidades de cuidados intensivos.
En México, el servicio en donde los pacientes pediátricos con asma grave deben ser atendidos por antonomasia es el de urgencias, donde se forman y trabajan tanto especialistas en pediatría como en urgencias, los cuales deben tener el juicio clínico necesario para otorgar una atención de calidad que limite el desarrollo de complicaciones.

\section{Enfoque teórico}

La educación médica, al igual que la que se imparte prácticamente en cualquier ámbito, suele abordarse con un predominio de la tendencia pasivo-receptiva, en la cual suele seguirse un modelo reproductor que genera licenciados que no cuentan con una verdadera capacidad de reflexión y que en su práctica profesional tienden al conformismo, a la rutina, con escaso interés en continuar con la preparación y resolver las situaciones diarias a las que se enfrenta [13].

Conciente de estas limitaciones se han desarrollado diferentes alternativas que exigen una participación más consciente e interesada por parte de residentes y docentes y con las que se pretende lograr un licenciado más pleno y transformador [14].

Las evaluaciones tradicionales no son útiles para explorar este tipo de práctica educativa porque sólo abordan los aspectos memorísticos y habilidades y destrezas simples y de corto alcance [13-15].

Lograr evaluar la capacidad del médico para llegar a la solución de problemas clínicos es una preocupación continua de los educadores, aún no logrado por quienes se dedican a la formación de médicos; este deseo es todavía mayor refiriéndose a los médicos que tienen la responsabilidad directa del cuidado de los pacientes [16].

Dentro de estos sistemas de evaluación alternativos y que se ubican dentro de la teoría participativa de la educación se encuentran las denominadas 'aptitudes clínicas', las cuales se definen como la capacidad de afrontar apropiadamente situaciones clínicas de un problema. La prestancia en la solución de situaciones clínicas problemáticas es una cualidad que se refiere al perfeccionamiento constante para poder influir favorablemente en las condiciones de salud, y constituye el estudio ordenado de las diversas manifestaciones 
de la enfermedad con el propósito de precisar un diagnóstico, aplicar un tratamiento, establecer un pronóstico e instituir medidas preventivas para la atención apropiada del paciente. El desarrollo de esta aptitud se basa en casos reales por medio de la observación clínica, el análisis documental y la crítica de las evidencias [13-17].

La aptitud tiene por objeto la evaluación y elección de los individuos en cuanto a su capacidad para una profesión, utilizando procedimientos psicotécnicos y de psicología aplicada, que lo lleva a la reflexión como elemento constitutivo de la construcción del conocimiento. La formación académica de los médicos, con tendencia hacia la posición consumista más que una actitud activa y participativa, y que es como se desempeñan la mayoría de ellos, prima la información obtenida para memorizar sin la reflexión o crítica del conocimiento [12-17].

En múltiples estudios se informa de los logros al evaluar el grado de aptitudes clínicas que, ante diferentes patologías (hipertensión, diabetes, traumatismo, etc.), han desarrollado tanto estudiantes de medicina como residentes de diferentes especialidades (ortopedia, anestesiología, medicina familiar, rehabilitación), instrumentos en donde se encontró que estas aptitudes suelen ser deficientes [12-18].

Tanto el médico residente de pediatría como el de urgencias debe tener una preparación que cuente con una postura participativa en la educación para desarrollar la capacidad de detección y la resolución de problemas clínicos, desde la más fidedigna obtención de datos y registro de los datos clínicos hasta la interpretación adecuada de los efectos del tratamiento y la actualización de los conocimientos más recientes de la enfermedad.

En el ámbito de urgencias se han publicado dos artículos que establecen que las aptitudes clínicas de los residentes de urgencias son bajas en el abordaje de pacientes traumatizados y que el microambiente laboral no parece ser muy propicio para desarrollar un proceso educativo participativo [19,20].

Dentro de los programas académicos y de los ámbitos clínicos por donde rotan los residentes de las especialidades de urgencias y pediatría se contempla el estudio de la agudización de pacientes con asma, pero hasta el momento no se cuenta con un estudio en donde se evalúe el abordaje de pacientes con asma grave y se com-
Tabla I. Distribución por grado académico y especialidad de los 41 residentes evaluados sobre aptitud clínica ante el paciente con asma grave.

\begin{tabular}{lccc}
\hline & Grado & $n$ & $\%$ \\
\hline Pediatría & 1. $^{\text {er }}$ año & 10 & 24,39 \\
\hline Urgencias & 1. ${ }^{\text {r }}$ año & 11 & 26,82 \\
\cline { 2 - 4 } & 2. ${ }^{\circ}$ año & 9 & 21,95 \\
\hline & er año & 11 & 26,82 \\
\hline
\end{tabular}

pare el grado de aptitudes desarrolladas por los médicos ante esta entidad.

\section{Sujetos y métodos}

Estudio observacional del tipo transversal, descriptivo, autorizado por el comité local de investigación, en el que se planteó evaluar, a través de un cuestionario elaborado a propósito, las aptitudes clínicas en el tratamiento de pacientes pediátricos con asma grave.

El instrumento de evaluación se construyó a través de cinco casos clínicos reales de pacientes pediátricos con asma grave. Para la validación de contenido y conceptual se sometió a dos rondas de expertos en pediatría e investigación educativa. Se emplearon los ítems con un consenso de 5 sobre 5 .

La aptitud clínica se consideró como la capacidad de detección y resolución de problemas clínicos reales, desde la obtención de los datos clínicos y su interpretación adecuada hasta la toma de decisiones terapéuticas, llegando a una reflexión y generando un criterio propio, explorada a través de los siguientes indicadores:

- Reconocimiento de indicios clínicos y paraclínicos: capacidad de reconocer la vinculación de los datos clínicos durante el interrogatorio, la exploración física y los resultados de estudios de laboratorio, con ciertas sospechas diagnósticas.

- Integración diagnóstica clínica: capacidad de integración de signos y síntomas por medio de hipótesis diagnósticas sindrómicas y nosológicas congruentes.

- Omisión con efectos iatrogénicos: no realizar ac- 
Tabla II. Distribución del número de ítems por indicadores, del instrumento para evaluar las aptitudes clínicas en pacientes con asma grave.

\begin{tabular}{lccc}
\hline Indicador & N. o de ítems & $\%$ \\
\hline Reconocimiento de indicios clínicos y paraclínicos & 20 & 13,33 \\
\hline Integración diagnóstica clínica & 26 & 17,33 \\
\hline Omisión con efectos iatrogénicos & 15 & 10,00 \\
\hline Comisión con efectos iatrogénicos & 15 & 10,00 \\
\hline Jerarquización de la patología & 15 & 10,00 \\
\hline Uso global de medios diagnósticos & 15 & 10,00 \\
\hline Uso global de recursos terapéuticos & 15 & 10,00 \\
\hline Crítica al colega de acciones previas & 10 & 6,66 \\
\hline Nivel de resolución de urgencias & 19 & 12,66 \\
\hline Total & 150 & 100 \\
\hline
\end{tabular}

Tabla III. Comparación de las medianas de las calificaciones por grado académico, de los 41 residentes evaluados sobre aptitudes clínicas en el abordaje de pacientes pediátricos con asma grave.

\begin{tabular}{llr}
\hline \multicolumn{1}{c}{ Grado } & Mediana (rango) \\
\hline Pediatría & Residente de 1. er año $(n=10)$ & $85(41-116)$ \\
\cline { 2 - 3 } & Residente de 1. er año $(n=11)$ & $90(44-114)$ \\
\hline$p=$ no significativa; Kruskal-Wallis. & Residente de 2. ${ }^{\circ}$ año $(n=9)$ & $92(58-124)$ \\
\hline
\end{tabular}

ciones que resulten indispensables, con el consiguiente perjuicio para el paciente.

- Comisión con efectos iatrogénicos: efectuar acciones innecesarias que resulten perjudiciales para el paciente.

- Jerarquización de la patología: capacidad de identificar e intervenir ante una patología en estado agudo.

- Uso global de medios diagnósticos: indicación y empleo de los medios para el diagnóstico, incluyendo la omisión y la comisión iatrogénicas.

- Uso global de recursos terapéuticos: indicación y empleo de los medios terapéuticos incluyendo la omisión y la comisión iatrogénicas.

- Crítica al colega de acciones previas: capacidad de reconocer si las acciones ejecutadas en un momento fueron pertinentes y adecuadas.

- Nivel de resolución de urgencias: acciones pertinentes de ingreso y alta de pacientes, así como de traslado posterior a estabilización o resolución de proceso agudo.

La versión final del instrumento quedó en 150 ítems y correspondió a un cuestionario de tipo 'verdadero', 'falso' y 'no sé, en el cual una respuesta correcta valía un punto, una respuesta incorrecta restaba un punto y una respuesta tipo 'no sé' equivalía a cero. La calificación se obtuvo restando el número de respuestas incorrectas al número de respuestas correctas. 
Tabla IV. Aptitud clínica en el abordaje del paciente pediátrico con asma grave en 41 residentes de las especialidades de pediatría y urgencias.

\begin{tabular}{|c|c|c|c|c|c|c|c|c|}
\hline & & Pediatría & Urgencias & & & cias & & \\
\hline & Rango & $\begin{array}{l}\text { Residentes } \\
\text { de } 1 .{ }^{\text {er }} \text { año }\end{array}$ & $\begin{array}{l}\text { Residentes } \\
\text { de } 1 .^{\text {er año }}\end{array}$ & $p^{a}$ & $\begin{array}{l}\text { Residentes } \\
\text { de } 2 .^{\circ} \text { año }\end{array}$ & $\begin{array}{l}\text { Residentes } \\
\text { de } 3 \text {. }^{\text {r }} \text { año }\end{array}$ & $p^{b}$ & $\%$ \\
\hline $\begin{array}{l}\text { Explicable } \\
\text { por azar }\end{array}$ & $0-25$ & & 0 & NS & 0 & 0 & NS & 0 \\
\hline Muy baja & $26-50$ & 3 & 6 & NS & 3 & 4 & NS & 16 \\
\hline Baja & $51-75$ & 6 & 3 & NS & 2 & 2 & NS & 13 \\
\hline Intermedia & 76-100 & 1 & 2 & NS & 4 & 5 & NS & 12 \\
\hline Alta & $101-125$ & 0 & 0 & NS & 0 & 0 & NS & 0 \\
\hline Muy alta & $126-150$ & 0 & 0 & NS & 0 & 0 & NS & 0 \\
\hline Total & & 10 & 11 & NS & 9 & 11 & NS & 100 \\
\hline
\end{tabular}

${ }^{\mathrm{a}} U$ de Mann-Whitney; ${ }^{\mathrm{b}}$ Kruskal-Wallis. NS: no significativa.

Se analizaron además las variables de especialidad, grado académico, rotación previa por el servicio de urgencias y pediatría, y realización de guardia el día inmediato anterior.

La prueba piloto se realizó a 20 residentes de pediatría y urgencias de una sede ajena al estudio; de acuerdo con la fórmula 21 de KuderRichardson, la consistencia fue de 0,89 . Según la prueba de Pérez-Padilla, las respuestas estimadas al azar resultaron 25 .

El instrumento se aplicó a todos los residentes de urgencias y pediatría que aceptaron participar en una determinada fecha. Se incluyó a 31 residentes de los tres grados de la especialidad de urgencias y a 10 residentes de primer año de pediatría (Tabla I).

Se empleó un muestreo no probabilístico por conveniencia en el que se incluyó a 31 residentes de urgencias y 10 de pediatría de un hospital de segundo nivel y que, tras ser informados de las características del estudio, aceptaron participar y respondieron al $100 \%$ el instrumento de evaluación.

Para el análisis de los datos se empleó estadística no paramétrica con pruebas como la de Kruskal-Wallis para estimar diferencias entre más de dos grados académicos y la prueba $U$ de Mann-Whitney para estimar diferencias entre dos grados académicos, rotación o no por urgencias-pediatría.

\section{Resultados}

Se evaluaron 150 ítems que abordan siete indicadores (Tabla II).

En las comparaciones de las medianas de los diferentes grados académicos, la prueba de Kruskal-Wallis no encontró diferencias significativas entre los grados académicos (Tabla III).

En el 70,73\% de los residentes se encontraron aptitudes bajas o muy bajas, en tanto que sólo el $28,27 \%$ se ubicaron en el rango intermedio. Ninguno alcanzó niveles altos o muy altos. La prueba de Kruskal-Wallis no encontró diferencias entre los niveles académicos, en tanto que la $U$ de Mann-Whitney no halló diferencias entre la especialidad (Tabla IV).

No se hallaron diferencias significativas en el análisis por los diferentes indicadores, tanto en lo referente al grado académico o la especialidad, como a la rotación previa por el servicio de urgencias-pediatría o el haber realizado guardia el día anterior (Tabla V).

\section{Discusión}

El asma y su agudización, la crisis asmática, son un padecimiento frecuente en los servicios de 
Tabla V. Comparación, por grado académico, de las medianas de los diferentes indicadores para evaluar la aptitud clínica en el abordaje de pacientes pediátricos con asma grave $(n=41)$.

\begin{tabular}{|c|c|c|}
\hline Indicador & Grado & Medianas \\
\hline Reconocimiento de indicios clínicos y paraclínicos & $\begin{array}{l}\text { 1. er año pediatría } \\
\text { 1.'r año urgencias } \\
\text { 2. }{ }^{\circ} \text { año urgencias } \\
\text { 3. } .^{\text {r }} \text { año urgencias }\end{array}$ & $\begin{array}{l}5 \\
8 \\
8 \\
9\end{array}$ \\
\hline Integración diagnóstica clínica & $\begin{array}{l}\text { 1. er año pediatría } \\
\text { 1. er año urgencias } \\
\text { 2. }{ }^{\circ} \text { año urgencias } \\
\text { 3. } .^{\text {er }} \text { año urgencias }\end{array}$ & $\begin{array}{l}10 \\
12 \\
14 \\
16\end{array}$ \\
\hline Omisión con efectos iatrogénicos & $\begin{array}{l}\text { 1. er año pediatría } \\
\text { 1.er año urgencias } \\
\text { 2. }{ }^{\circ} \text { año urgencias } \\
\text { 3. } .^{\text {er }} \text { año urgencias }\end{array}$ & $\begin{array}{l}3 \\
4 \\
6 \\
5\end{array}$ \\
\hline Comisión con efectos iatrogénicos & $\begin{array}{l}\text { 1. er año pediatría } \\
\text { 1.er año urgencias } \\
\text { 2. }{ }^{\circ} \text { año urgencias } \\
\text { 3. } .^{\text {er }} \text { año urgencias }\end{array}$ & $\begin{array}{l}3 \\
4 \\
5 \\
6\end{array}$ \\
\hline Jerarquización de la patología & $\begin{array}{l}\text { 1. er año pediatría } \\
\text { 1. er año urgencias } \\
\text { 2. }{ }^{\circ} \text { año urgencias } \\
\text { 3. er año urgencias }\end{array}$ & $\begin{array}{c}8 \\
9 \\
9 \\
11\end{array}$ \\
\hline Uso global de medios diagnósticos & $\begin{array}{l}\text { 1. er año pediatría } \\
\text { 1. er año urgencias } \\
\text { 2. }{ }^{\circ} \text { año urgencias } \\
\text { 3. } .^{\text {er }} \text { año urgencias }\end{array}$ & $\begin{array}{l}4 \\
4 \\
6 \\
7\end{array}$ \\
\hline Uso global de recursos terapéuticos & $\begin{array}{l}\text { 1. er año pediatría } \\
\text { 1.' año urgencias } \\
\text { 2. }{ }^{\circ} \text { año urgencias } \\
\text { 3. } .^{\text {er }} \text { año urgencias }\end{array}$ & $\begin{array}{l}5 \\
5 \\
6 \\
8\end{array}$ \\
\hline Crítica al colega de acciones previas & $\begin{array}{l}\text { 1. er año pediatría } \\
\text { 1. er año urgencias } \\
\text { 2. }{ }^{\circ} \text { año urgencias } \\
\text { 3. } .^{\text {r }} \text { año urgencias }\end{array}$ & $\begin{array}{l}4 \\
5 \\
5 \\
4\end{array}$ \\
\hline Nivel de resolución de urgencias & $\begin{array}{l}\text { 1. er año pediatría } \\
\text { 1. er año urgencias } \\
\text { 2. }{ }^{\circ} \text { año urgencias } \\
\text { 3. er año urgencias }\end{array}$ & $\begin{array}{l}3 \\
3 \\
4 \\
6\end{array}$ \\
\hline
\end{tabular}

urgencias. El coste de su atención y la posibilidad de complicaciones también son elevados. El hecho de establecer si los futuros médicos especialistas que se enfrentarán a ella de forma cotidiana cuentan con la aptitud clínica necesaria resulta fundamental para poder establecer mo- dificaciones en las estrategias educativas con que se aborda esta entidad.

Si bien es cierto que el estudio de las aptitudes clínicas dentro de las especialidades médicas tiene ya algunos años en México, su abordaje dentro de urgencias se ha retrasado. En esto probable- 
mente influyen tanto lo reciente de la especialidad como la falta de formación docente de los profesores responsables en las diferentes sedes.

Haber elegido el asma como objeto de estudio tiene gran importancia, ya que esta patología resulta frecuente y suele requerir capacidades distintivas de otras especialidades, como una toma de decisiones más rápida, aunque no por ello menos reflexiva. Al contrario, merece un proceso de profunda interiorización, reflexión, crítica e integración de la información y las experiencias previas, al confrontarlas en situaciones reales y contextualizadas.

El proceso de validación fue largo e incluyó dos rondas de expertos; aportó una consistencia interobservador adecuada, con lo que se logró un instrumento que explora el grado de precisión del criterio individual (aptitud clínica) y un ajuste en la validez conceptual y de contenido. No obstante, este proceso es susceptible de perfeccionarse.

Las opciones de respuesta fueron 'verdadero', 'falso' y 'no se'; esta última es de vital importancia porque permite al residente hacerse consciente de sus limitaciones al estar seguro de que ello no repercutirá en su evaluación final.

Los problemas de la práctica clínica mediante casos reales confieren una significativa relevancia, ya que permiten valorar el alcance de las decisiones y los criterios médicos bajo las diferentes circunstancias a las que se enfrenta el residente en su práctica diaria.

Que en este estudio haya participado el 88\% de los residentes de la especialidad de urgencias con sede en el Distrito Federal permiten llevar con cierta fiabilidad los resultados a la totalidad de los mismos.

No sorprende haber encontrado que en la mayoría de los residentes se presentaron aptitudes dentro de los niveles inferiores, ya que precisamente esta falta de proceso reflexivo de la práctica diaria rutinaria se genera con las estrategias educativas con orientación pasivo-receptiva, que actualmente priman en las sedes y que sólo favorecen aspectos memorísticos, repetitivos y poco motivadores para la participación.

La experiencia clínica que supuestamente el proceso formativo debería lograr con el paso de los años de residencia cursados, no parece tener un efecto notorio en las aptitudes clínicas obtenidas por los residentes, lo que traduce una discrepancia entre la teoría (capacidades que debe tener) y la práctica (capacidades que en realidad tiene).
La aptitud clínica del médico residente de urgencias y el de pediatría en el conocimiento y manejo del paciente pediátrico con crisis de asma es deficiente, y no existen diferencias en las aptitudes clínicas en relación al nivel académico o la rotación previa por el servicio de urgenciaspediatría.

En conclusión, los resultados sugieren que el proceso educativo al que se han sometido estos residentes a lo largo de toda su formación académica, parece sostenerse sobre la pasividad tradicional de la escuela.

No se ha desarrollado en estos residentes un criterio propio, lo que limitará de forma importante la solución que deba adoptar ante problemas reales durante su práctica profesional. Por tanto, será necesario establecer estrategias y proyectos educativos que indaguen otras formas de desarrollar el proceso educativo.

\section{Bibliografía}

1. National Asthma Education and Prevention Program. Guidelines for the diagnosis and management of asthma. Bethesda: US Department of Health and Human Services. NIH Publication 97-4051; 1997.

2. Alexander HL. An historical account of death from asthma. J Allergy 1963; 34: 305-13.

3. Altman LK The public perception of asthma. In Sheffer AL, ed. Fatal asthma. New York: Marcel Dekker; 1998. p. 1-11.

4. Global Initiative for Asthma (GINA). Pocket guide for asthma management and prevention. Bethesda: US Department of Health and Human Services. Public Health Services, National Institutes of Heart, Kung, and Blood Institute; 2002.

5. Toelle BG, Peat JK, Salomé CM, Mellis CM Woolcock D. Toward a definition of asthma epidemiology. Am Rev Dis 1992; 146: 633-7.

6. Beasley RK, Mutius EV, Pearce N. Worldwide variation in prevalence of symptoms of asthma, allergic rhinoconjuntivitis, and atopia eczema ISSAC. The International Study of Asthma and Allergies in Childhood (ISAAC) Steering Committee. Lancet 1998; 351: 1225-32.

7. Silver EJ, Crain EF, Weiss KB. Burden of wheezing illness among US. Children reported by parents not to have asthma. J Asthma 1998; 35: 437-43.

8. Boner AL, Mortinati LC. Diagnosis of asthma in children and adolescents. Eur Resp Rev 1997; 7: 3-7. 
9. Romieu I, Hernández-Ávila M. Effects of urban air pollutants of emergency visits for childhood asthma in México City. Am J Epidemiol 1995; 141: 546-53.

10. Tatto-Cano MI, Sanin-Aguirre LH, González V. Prevalencia del asma, rinitis y eczema en escolares de la ciudad de Cuernavaca, Sal Pub Mex 1997; 39: 496-506.

11. Sierna-Monge JJ, Romieu I. Suplementación con antioxidantes y salud respiratoria en niños asmáticos expuestos a altos niveles de contaminantes. Encuentro Nacional de Investigadores en salud, San Juan del Río, Querétaro, 2001.

12. Instituto Nacional de Enfermedades Respiratorias. Coordinación de Institutos Nacionales de Salud. Secretaría de Salud. Informe de labores 2000-2001.

13. Viniegra L, Jiménez JL, Pérez-Padilla JR. El desafío de la evaluación en la competencia clínica. Rev Invest Clin 1991; 43: 87-97.

14. Viniegra L. La formación de médicos familiares y el desarrollo de la aptitud clínica. Rev Med IMSS 2004; 42: 309-20.
15. Viniegra L, García H, Briceño A, Carrillo R, Gómez L, Herrera M. Utilidad comparativa de dos tipos de exámenes empleados con fines selectivos. Rev Invest Clin 1985; 37: 253-6.

16. Sabido C, Viniegra L. Competencia y desempeño clínicos en diabetes. Rev Invest Clin 1998; 50: 211-6.

17. Arnaiz TC, Rodríguez PS, Mercado MR. Evaluación de las estrategias en la formación de especialistas en el Instituto Mexicano del Seguro Social. Rev Med IMSS 1994; 32: 187-90.

18. Rivera DB, Aguilar E, Viniegra VL. Evaluación de la aptitud clínica de médicos residentes de medicina física y rehabilitación. Rev Invest Clin 1998; 50: 341-6.

19. Loría-Castellanos J, Rivera-Ibarra DB. Aptitudes clínicas de residentes de urgencias médicas en el manejo de patología traumática. Rev Med IMSS 2005; 43: 17-23.

20. Chavarría R. Rivera D. Entorno laboral y aptitudes clínicas en residentes de urgencias médico quirúrgicas. Rev Med IMSS 2004; 42: 337-80. 